\title{
Transmit Antenna Subset Selection in Generalised Spatial Modulation Systems
}

\author{
Rakshith Rajashekar, Senior Member, IEEE, Lie-Liang Yang, Fellow, IEEE, K.V.S. Hari, Fellow, IEEE and \\ L. Hanzo, Fellow, IEEE
}

\begin{abstract}
The existing literature on transmit antenna selection (TAS) aided spatial modulation (SM) systems extensively deals with the Euclidean distance (ED) based TAS owing to its high transmit diversity gain. In this paper, we consider the EDTAS aided generalized spatial modulation (GSM) system that transmits multiple symbols in each channel use and characterise its attainable diversity gain. Secondly, we show that the minimum ED of the receive constellation does not grow beyond a certain point when the size of the signal set is sufficiently large, which facilitates low-complexity implementations of the ED-TAS aided SM system. Our numerical studies revealed that a signal-to-noise gain of about $8 \mathrm{~dB}$ is achievable by employing ED-TAS in the GSM system compared to its counterpart dispensing with EDTAS.
\end{abstract}

Index Terms-Antenna subset selection, generalised spatial modulation, diversity gain, lattice, SWIPT.

\section{INTRODUCTION}

The next generation communication systems are not only expected to be spectrally efficient but also have high energy efficiency. In order to cater for the latter requirement, several energy efficient modulation schemes were proposed in the recent past, which include spatial modulation (SM), space shift keying (SSK), generalised spatial modulation (GSM), index modulation (IM) [1]-[7] etc. A key feature of these schemes is that they require only a single RF-chain or at least a low number of them at the transmitter in comparison to the conventional MIMO systems [8]. This in turn significantly reduces the transmitter's power consumption. Furthermore, since only a single or a few antennas are activated, this mitigates the inter channel interference (ICI), which makes them amenable for low-complexity maximum likelihood (ML) detection [4].

Since the number of active transmit antennas (TAs) is restricted, they suffer from the lack of transmit diversity. Several open-loop [9]-[12] and closed-loop techniques [13][24] were conceived in order to overcome this impediment. The closed-loop techniques can be broadly classified into a)

Copyright (c) 2015 IEEE. Personal use of this material is permitted. However, permission to use this material for any other purposes must be obtained from the IEEE by sending a request to pubs-permissions@ieee.org. R. Rajashekar, L-L. Yang and L. Hanzo are with the School of ECS, University of Southampton, SO17 1BJ, UK (e-mail: rmr1u14@soton.ac.uk, 1ly@ecs.soton.ac.uk, lh@ecs.soton.ac.uk). K.V.S. Hari is with the Dept. of ECE, Indian Institute of Science, Bangalore, 560012, India (e-mail: hari@ece.iisc.ernet.in).

The financial support of the EPSRC projects EP/P034284/1, EP/N004558/1 and EP/L018659/1, as well as of the ERC's Advanced Fellow Award QuantCom is gratefully acknowledged. All data supporting this study are openly available from the University of Southampton repository at https://doi.org/10.5258/SOTON/D0704. modulation-order selection [13], b) TA-subset selection [14][24]. In [13], Yang et al. studied a link-adaptive scheme based on modulation order selection, while both capacity based and Euclidean distance (ED) based transmit antenna selection (EDTAS) schemes were proposed in [14]. Furthermore, their performances were evaluated under imperfect channel conditions in [15] and their low-complexity counterparts were proposed in [16], [17]. In [19], Sun et al. proposed a cross-entropy based method for reducing the search complexity of ED-TAS, while the authors of [18] quantified the transmit diversity order of ED-TAS. An improved low-complexity implementation of ED-TAS that strikes a beneficial performance vs. complexity trade-off was proposed in [20], while Sun et al. [21] conceived a reduced-dimensional ED-TAS-equivalent criterion, which results in the same performance as that of ED-TAS, despite its reduced complexity. In [22], Naresh et al. studied the ED based mirror activation pattern selection schemes in the context of RF-mirror aided spatial modulation systems. A generalized transmit and receive diversity condition was proposed in [23] for MIMO systems based on the ED metric, while ED-TAS was studied in frequency selective channels for the first time in [24]. In [25], the authors have studied the benefits of TAS in secure communication in the presence of an eavesdropper. Recently, the authors in [26], [27] have proposed an opportunistic antenna selection based on antenna grouping and evaluated the closed form expression for the union-bound on the symbol error probability.

Against this background, the following are the contributions of this paper:

1) The existing TA-subset selection algorithms [14]-[24] were mainly conceived for SM systems, where only a single TA is activated in each channel use. In this paper, we consider the generalized spatial modulation (GSM) system [3] employing ED-TAS, where multiple TAs are activated simultaneously. Furthermore, we quantify the attainable diversity order of the GSM system employing ED-TAS and show that it attains the same transmit diversity gain as that of the SM system employing EDTAS. In other words, our results show that the high diversity gain attained by the SM system employing ED-TAS [18] is retained by the GSM system as well, thus providing sufficient motivation to look for lowcomplexity solutions to ED-TAS aided GSM systems in the future.

2) A key bottleneck in ED-TAS schemes is the computation of the minimum ED over the signal set for each of the 
antenna combinations. The computational complexity of this metric grows with $M^{2}$ in case of the SM system, where $M$ is the size of the signal set. Thus, when $M$ is large, the computational complexity may be formidable. We show that the minimum ED does not change when the size of the signal set is increased beyond a certain value. As a result, the optimum TA subset remains the same, when the size of the signal set is increased. This enables us to compute the ED metric by assuming moderately large signal sets and use the optimal TA combination obtained in conjunction with larger signal sets, thus avoiding the computation of the minimum ED over extremely large signal sets.

The remainder of the paper is organized as follows. Our results on the GSM system employing ED-TAS are described in Section II. The characterisation of the trade-off between the harvested energy and the minimum ED in the SM system employing ED-TAS is provided in Section III. Finally, Section IV concludes the paper.

\section{GSM SYSTEM EMPLOYING ED-TAS}

\section{A. System Model}

Consider a GSM system having $N_{t}$ TAs, $N_{r}$ receive antennas (RAs) and $N_{a}$ transmit RF-chains, whose system model is given by

$$
\mathbf{y}=\sqrt{\frac{\rho}{N_{a}}} \mathbf{H s}+\mathbf{n},
$$

where $\mathbf{y} \in \mathbb{C}^{N_{r}}$ is the received vector, $\rho$ is the average signalto-noise power ratio (SNR) at each $\mathrm{RA}, \mathbf{H} \in \mathbb{C}^{N_{r} \times N_{t}}$ is the channel matrix, $\mathbf{s} \in \mathbb{C}^{N_{t}}$ is the transmit vector having only $N_{a}$ non-zero entries, $\mathbf{n} \in \mathbb{C}^{N_{r}}$ is the noise vector. The entries of $\mathbf{H}$ and $\mathbf{n}$ are from a circularly symmetric complex-valued Gaussian distribution $\mathcal{C N}(0,1)$. The non-zero entries of $\mathbf{s}$ are from a unit-energy $M$-QAM or $M$-PSK constellation, which is represented by $S$. The rate achieved by the GSM system is given by

$$
R_{G S M}=\log _{2}\left\lfloor\left(\begin{array}{c}
N_{t} \\
N_{a}
\end{array}\right)\right\rfloor_{2}+N_{a} \log _{2} M \text { bpcu, }
$$

where bpcu stands for bits per channel use and $\lfloor\cdot\rfloor_{2}$ represents flooring to the nearest power of two.

ED-TAS: Let $N_{S M}$ out of $N_{t}$ antennas be chosen for spatial modulation based on the minimum ED criterion. Let the enumerations of $n=\left(\begin{array}{c}N_{t} \\ N_{S M}\end{array}\right)$ combination of antennas be represented by the set $\mathcal{I}=\left\{I_{1}, I_{2}, \ldots, I_{n}\right\}$. The optimal TA subset chosen based on the minimum ED criterion is given by

$$
I^{*}=\arg \max _{I \in \mathcal{I}} \min _{\mathbf{s}_{1} \neq \mathbf{s}_{2}}\left\|\mathbf{H}_{I}\left(\mathbf{s}_{1}-\mathbf{s}_{2}\right)\right\|^{2},
$$

where $\mathbf{H}_{I} \in \mathbb{C}^{N_{r} \times N_{S M}}$ corresponds to the $N_{S M}$ columns given by $I$ and $\mathbf{s}_{i} \in \mathcal{X}$ for $i=1,2$, where $\mathcal{X}$ is the set of all legitimate transmit GSM vectors. Upon obtaining $I^{*}$, the receiver encodes this information and sends it to the transmitter. Upon receiving $I^{*}$, the transmitter uses the TAs indexed by $I^{*}$ for the transmission of the GSM signal.
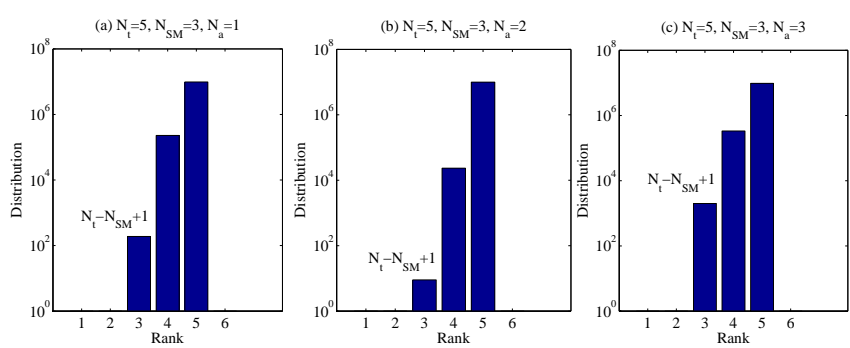

Fig. 1. Comparison of the distribution of $\operatorname{rank}(\mathbf{X})$ for $10^{7}$ samples of $\mathbf{X} \in \Delta \mathcal{D}$ in a GSM system having $N_{t}=5, N_{S M}=3$, and $N_{a} \in\{1,2,3\}$.

\section{B. Main Results}

Let $G_{i}$ represent the set of spatial modulation indices associated with the GSM vectors in conjunction with the TA combination $I_{i}$. For example, in a GSM system having $N_{t}=4, N_{S M}=3$ and $N_{a}=2$, we have $I_{1}=$ $\{1,2,3\}, I_{2}=\{1,2,4\}, I_{3}=\{1,3,4\}, I_{4}=\{2,3,4\}$ and $G_{1}=\{(1,2),(2,3)\}, G_{2}=\{(1,2),(2,4)\}, G_{3}=$ $\{(1,3),(3,4)\}, G_{4}=\{(2,3),(3,4)\}$.

Let $\mathcal{C}_{i}=\left\{\sum_{k \in J} s_{k} \mathbf{e}_{k} \mid s_{k} \in S, J \in G_{i}\right\}$ for $1 \leq i \leq n$ represent the sets of GSM transmit vectors corresponding to the $n$ antenna subsets, where $\mathbf{e}_{k}$ is the $k^{\text {th }}$ column of $N_{t} \times N_{t}$ identity matrix. Let $\Delta \mathcal{C}_{i}=\left\{\mathbf{x}_{1}-\mathbf{x}_{2} \mid \mathbf{x}_{1}, \mathbf{x}_{2} \in \mathcal{C}_{i}, \mathbf{x}_{1} \neq \mathbf{x}_{2}\right\}$ represent the set of difference vectors corresponding to the code book $\mathcal{C}_{i}$.

Lemma 1 (Proposition 1 [18]): The GSM system employing ED-TAS achieves a diversity gain of $N_{r} p$ under ML detection, where $p=\min \{\operatorname{rank}(\mathbf{X}) \mid \mathbf{X} \in \Delta \mathcal{D}\}$ and

$$
\Delta \mathcal{D}=\left\{\left[\mathbf{x}_{1}, \mathbf{x}_{2}, \ldots, \mathbf{x}_{n}\right] \mid \mathbf{x}_{1} \in \Delta \mathcal{C}_{1}, \ldots, \mathbf{x}_{n} \in \Delta \mathcal{C}_{n}\right\} .
$$

Note that the attainable transmit diversity order of the GSM system employing ED-TAS is given by $p$.

Proposition 1: The transmit diversity order of the GSM system employing ED-TAS is the same as that of the SM system employing ED-TAS, i.e.

$$
p=\min \{\operatorname{rank}(\mathbf{X}) \mid \mathbf{X} \in \Delta \mathcal{D}\}=N_{t}-N_{S M}+1
$$

and is independent of $N_{a}$.

Proof: Let $\Delta \mathcal{D}_{S M}$ represent the set of matrices of difference vectors associated with the SM system employing EDTAS, i.e. $\Delta \mathcal{D}_{S M}$ corresponds to $\Delta \mathcal{D}$ in (4) with $N_{a}=1$. It is readily seen that there exists an $\mathbf{X} \in \Delta \mathcal{D}_{S M}$ ensuring that $\operatorname{rank}(\mathbf{X})=N_{t}-N_{S M}+1$, which also belongs to $\Delta \mathcal{D}$ of the GSM system given in (4). Thus, we have $p \leq N_{t}-N_{S M}+1$. Since any $\mathbf{X} \in \Delta \mathcal{D}$ cannot be partitioned into less than $N_{t}-N_{S M}+1$ sets of columns, where the elements in each set are linearly independent of the elements in the other sets, we have $p \geq N_{t}-N_{S M}+1$. Thus, equality follows, i.e. $p=N_{t}-N_{S M}+1$.

Remark 1: Note that the TAS aided V-BLAST system attains a transmit diversity order of $N_{t}-N^{\prime}+1$ [28], where $N^{\prime}<N_{t}$ is the number of spatial data streams. This corresponds to the special case of $N_{S M}=N_{a}=N^{\prime}$ in our ED-TAS aided GSM framework. Furthermore, our diversity analysis is based on the minimum ED of the receive signal 
constellation while assuming ML detection at the receiver, whereas that of [28] is based on the geometrical framework that assumes linear and decision-feedback receivers.

Let us now characterise our result in Proposition 1 by numerical studies. Consider a GSM system employing ED-TAS and having $N_{t}=5, N_{S M}=3, N_{a} \in\{1,2,3\}$, employing a BPSK signal set. Fig. 1 provides the distribution of $\operatorname{rank}(\mathbf{X})$ for $10^{7}$ samples of $\mathbf{X} \in \Delta \mathcal{D}$ in the aforementioned GSM system for three values of $N_{a} \in\{1,2,3\}$. It is evident from Fig. 1 that $\min \{\operatorname{rank}(\mathbf{X}) \mid \mathbf{X} \in \Delta \mathcal{D}\}=N_{t}-N_{S M}+1$ for each value of $1 \leq N_{a} \leq N_{S M}$. Thus, we conclude that the transmit diversity order of the GSM system employing EDTAS is the same as that of the SM system employing ED-TAS, and is independent of the value of $N_{a}$.

Let us now compare the bit error rate (BER) performance of the GSM system employing ED-TAS to that operating without ED-TAS. Consider a ED-TAS aided GSM system having $N_{a}=2, N_{S M}=3, N_{t} \in\{3,4,5\}, N_{r}=1$ and employing a BPSK signal set. Fig. 2 compares the BER performance of the aforementioned system for various values of $N_{t} \in\{3,4,5\}$. It is evident from Fig. 2 that the ED-TAS aided GSM system outperforms the system dispensing with TAS. Specifically, at a BER of $2 \times 10^{-3}$, an SNR gain of about $6 \mathrm{~dB}$ and $8 \mathrm{~dB}$ were observed in case of $N_{t}=4$ and $N_{t}=5$ compared to the $N_{t}=3$ scenario. Fig. 3 compares the BER performance of the aforementioned system employing a 4-QAM signal set. Again, it is evident from Fig. 3 that the ED-TAS aided GSM system outperforms the system operating without TAS. Specifically, at a BER of $10^{-2}$, an SNR gains of about $4 \mathrm{~dB}$ and $5 \mathrm{~dB}$ were observed in case of $N_{t}=4$ and $N_{t}=5$ compared to the $N_{t}=3$ scenario.

Fig. 4 depicts the variation in the BER performance of ED-TAS aided GSM system having $N_{a}=2, N_{S M}=3$, $N_{t}=\{3,4\}, N_{r} \in\{1,2,3\}$ and employing 4-QAM signal set. It is evident from Fig. 4 that the ED-TAS aided GSM outperforms its counterpart without TAS for various values of $N_{r}$. Fig. 5 compares the BER performance of the EDTAS aided GSM system to that of its SM counterpart when operating at 6 and $8 \mathrm{bpcu}$. Both the systems are assumed to have $N_{t}=5, N_{S M}=4, N_{r}=2$ and attain a transmit diversity order two. When operating at 6 and $8 \mathrm{bpcu}$, the GSM system employs 4-QAM and 8-QAM signal sets, while the SM system employs 16-QAM and 64-QAM signal sets, respectively. It is seen in Fig. 5 that the ED-TAS aided GSM system outperforms its SM counterpart at both the datarates. Specifically, at a BER of $10^{-4}$ the GSM system attains an SNR gain of about $3 \mathrm{~dB}$ and about $1 \mathrm{~dB}$ w.r.t. the SM system when operating at 6 and 8 bpcu, respectively. Fig. 6 compares the BER performance of the ED-TAS aided GSM system to that of its SM counterpart when $N_{r}=3$. The other system parameters are the same as those mentioned earlier. In this case, at a BER of $10^{-4}$ the GSM system attains an SNR gain of about $3 \mathrm{~dB}$ and about $4 \mathrm{~dB}$ w.r.t. the SM system when operating at 6 and $8 \mathrm{bpcu}$, respectively.

Remark 2: Although the ED-TAS aided GSM system attains significantly better performance compared to its counterpart using no TAS, the computational complexity imposed by it is excessive. A major complexity contributor is the

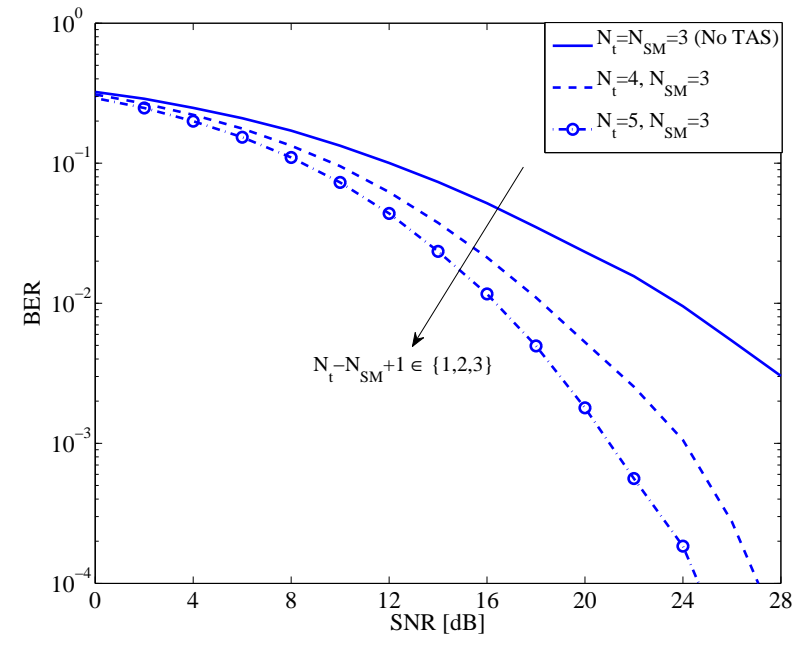

Fig. 2. Comparison of the BER performance of a ED-TAS aided GSM system having $N_{a}=2, N_{S M}=3, N_{t} \in\{3,4,5\}, N_{r}=1$ and employing BPSK signal set.

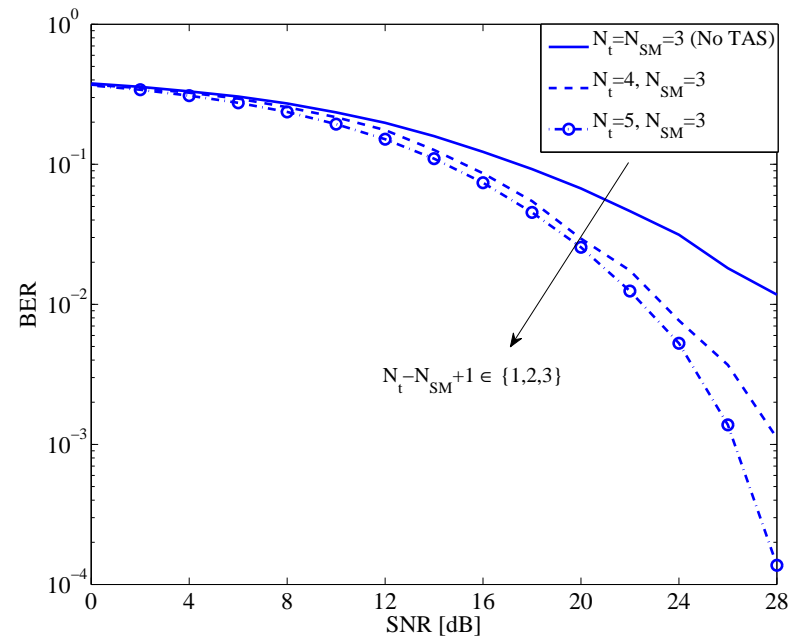

Fig. 3. Comparison of the BER performance of a ED-TAS aided GSM system having $N_{a}=2, N_{S M}=3, N_{t} \in\{3,4,5\}, N_{r}=1$ and employing 4-QAM signal set.

minimum ED computation in (3), which is over all possible transmit vectors. In a GSM system having $N_{a}$ active TAs, the complexity of the minimization problem in (3) is of the order $\left(M^{N_{a}}\left\lfloor\left(\begin{array}{c}N_{S M} \\ N_{a}\end{array}\right)\right\rfloor_{2}\right)^{2}$. Thus, it is an interesting open problem to conceive low-complexity solutions for reducing the computational burden imposed by (3).

Recall that the minimum ED in (3) is given by $\min _{\mathbf{s}_{1} \neq \mathbf{s}_{2}}\left\|\mathbf{H}_{I}\left(\mathbf{s}_{1}-\mathbf{s}_{2}\right)\right\|^{2}$, whose complexity grows with $M^{2 N_{a}}$. Considering the SM system where $N_{a}=1$, we show that the minimum ED does not change, when $M$ is increased beyond a certain value. The following proposition describes the above result. A similar argument holds for the case of the GSM system, where $N_{a}>1$.

Proposition 2: Let $\operatorname{MED}(M)=\min _{\mathbf{s}_{1} \neq \mathbf{s}_{2}}\left\|\mathbf{H}_{I}\left(\mathbf{s}_{1}-\mathbf{s}_{2}\right)\right\|^{2}$. 


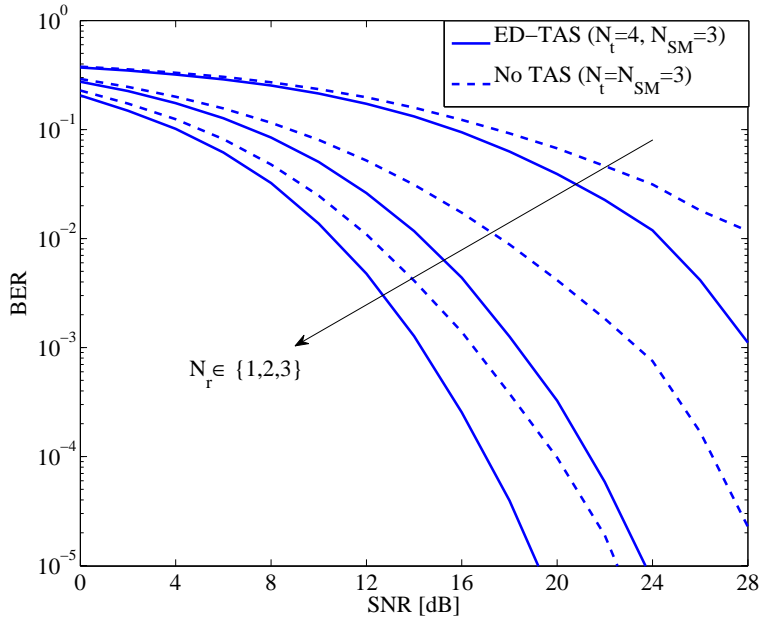

Fig. 4. Comparison of the BER performance of ED-TAS aided GSM with that without TAS in a system having $N_{a}=2, N_{S M}=3, N_{t}=\{3,4\}$, $N_{r} \in\{1,2,3\}$ and employing 4-QAM signal set.

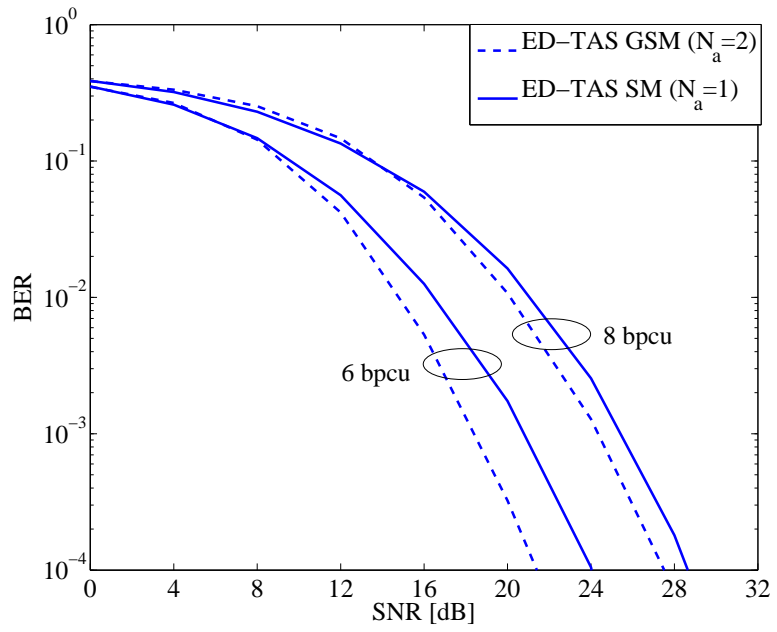

Fig. 5. Comparison of the BER performance of a ED-TAS aided GSM and its SM counterpart in a system having $N_{a} \in\{1,2\}, N_{S M}=4, N_{t}=5$, $N_{r}=2$ while operating at 6 and 8 bpcu.

For a given channel realization $\mathbf{H}$, we have

$$
\lim _{\left(M_{2}, M_{1}\right) \rightarrow \infty}\left|\operatorname{MED}\left(M_{2}\right)-\operatorname{MED}\left(M_{1}\right)\right|=0, M_{2}>M_{1} .
$$

Proof: Note that $\operatorname{MED}(M)=\min _{\mathbf{s}_{1} \neq \mathbf{s}_{2}}\left\|\mathbf{H}_{I}\left(\mathbf{s}_{1}-\mathbf{s}_{2}\right)\right\|^{2}=$ $\min \left\{\mathbf{D}^{(M)}(I)\right\}$, where $\mathbf{D}_{i, j}^{(M)}=\min _{s_{1}, s_{2} \in M-Q A M} \| \mathbf{h}_{i} s_{1}-$ $\mathbf{h}_{j} s_{2} \|^{2}$ for $j>i, \mathbf{D}_{i, j}^{(M)}=\min _{s_{1} \neq s_{2} \in M-Q A M}\left\|\mathbf{h}_{i}\right\|^{2}\left|s_{1}-s_{2}\right|^{2}$ for $j=i$ and zero otherwise [14], and $\mathbf{D}^{(M)}(I)$ represents a submatrix of $\mathbf{D}$ obtained by retaining the rows and columns indexed by $I$. It may be readily seen that when $j=i, \mathbf{D}_{i, j}^{\left(M_{2}\right)}=$ $\mathbf{D}_{i, j}^{\left(M_{1}\right)}$ for any $M_{2}>M_{1}$. When $j>i$, we have

$$
\begin{aligned}
\mathbf{D}_{i, j}^{(M)} & =\min _{s_{1}, s_{2} \in M-Q A M}\left\|\mathbf{h}_{i} s_{1}-\mathbf{h}_{j} s_{2}\right\|^{2}, \\
& =\min _{s_{1}, s_{2} \in M-Q A M}\left\|\left[\mathbf{h}_{i} \mathbf{h}_{j}\right]\left[\begin{array}{ll}
s_{1} & -s_{2}
\end{array}\right]^{T}\right\|^{2} .
\end{aligned}
$$

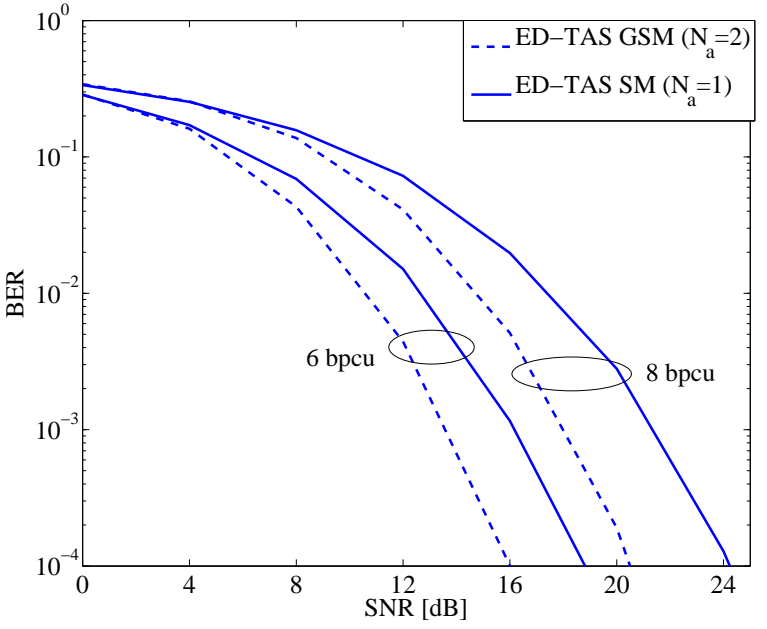

Fig. 6. Comparison of the BER performance of a ED-TAS aided GSM and its SM counterpart in a system having $N_{a} \in\{1,2\}, N_{S M}=4, N_{t}=5$, $N_{r}=3$ while operating at 6 and 8 bpcu.

By taking the QR decomposition [29] of the real-valued matrix of $\left[\mathbf{h}_{i} \mathbf{h}_{j}\right]$, we have

$$
\mathbf{D}_{i, j}^{(M)}=\min _{\substack{s_{1 I}, s_{1 Q} \in N_{1}-P A M \\
s_{2 I}, s_{2 Q} \in N_{2}-P A M}}\left\|\mathbf{R}\left[\begin{array}{c}
s_{1 I} \\
s_{1 Q} \\
-s_{2 I} \\
-s_{2 Q}
\end{array}\right]\right\|^{2},
$$

where $N_{1}$-PAM and $N_{2}$-PAM are the decompositions of the $M$-QAM along the real and imaginary axes and $\mathbf{R}$ is a $4 \times 4$ upper triangular matrix having zeros at $(3,4)$ and $(1,2)$. It is easy to see that the columns of $\mathbf{R}$ are linearly independent and hence the set $\left\{\mathbf{R s} \mid \mathbf{s} \in(2 \mathbb{Z}+1)^{4}\right\}$ is a discrete subset of $\mathbb{R}^{4}$ constituting a lattice. Thus, as $M \rightarrow \infty, N_{1}$ and $N_{2}$ become large and hence $\mathbf{D}_{i, j}^{(M)}$ converges to the minimum distance of the lattice. As a result, $\left|\operatorname{MED}\left(M_{2}\right)-\operatorname{MED}\left(M_{1}\right)\right| \rightarrow 0$ when $M_{2} \rightarrow \infty$ and $M_{1} \rightarrow \infty$. This concludes the proof.

Let us now illustrate the result in Proposition 2 by numerical studies. For the ease of presentation, let us denote $\mathbf{D}^{(M)}$ by $\mathbf{D}_{k}$, where $k=\log _{2} M$. Fig. 7 illustrates the variation in $\left\|\mathbf{D}_{k+1}-\mathbf{D}_{k}\right\|_{F}^{2}$ as a function of $k$ in an SM system having $N_{t}=6, N_{S M}=4$, and $N_{r} \in\{1,2,3\}$. It is evident from Fig. 7(a)-(c) that when $k$ is large, we have $\mathbf{D}_{k+1}=\mathbf{D}_{k}$. Furthermore, we see that the larger $N_{r}$, the lower the value of $k$ for which we have $\mathbf{D}_{k+1}=\mathbf{D}_{k}$. An important consequence of this result is that a moderate signal set size can be used for finding the optimal antenna subset and then the antenna subset obtained can be used for any larger signal sets without loss of optimality. This substantially reduces the computational cost of finding the optimal antenna subset for very large signal sets. For example, when $N_{r}=3$ the minimum distance spectrum in (8) does not change for $M>2^{3}$ as shown in Fig. 7. Thus, the search complexity in (8) does not grow beyond $M^{2}=2^{6}$ even for signal sets of size $M=2^{l}>2^{3}$. Thus, the reduction in the search complexity order is given by $2^{2 l}-2^{6}, l>3$. 
(a) $\mathrm{N}_{\mathrm{r}}=1$

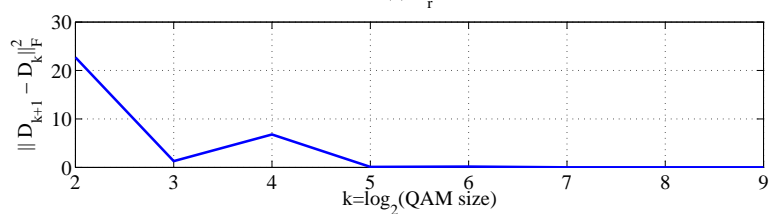

(b) $\mathrm{N}_{\mathrm{r}}=2$

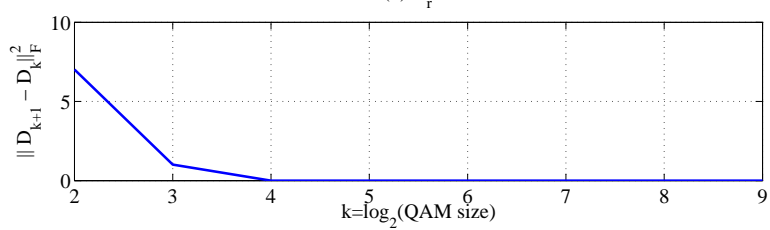

(c) $\mathrm{N}_{\mathrm{r}}=3$

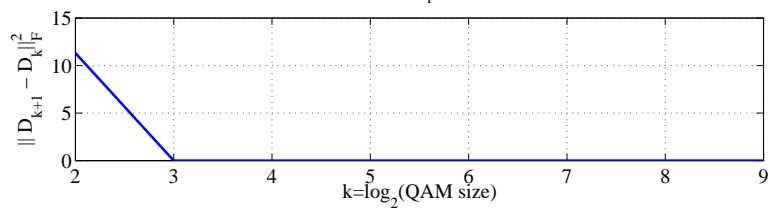

Fig. 7. Comparison of $\left\|\mathbf{D}_{k+1}-\mathbf{D}_{k}\right\|_{F}^{2}$ as a function of $k$ in an SM system having $N_{t}=6, N_{S M}=4$, and $N_{r} \in\{1,2,3\}$.

\section{CONCLUSions AND Future Work}

We have proposed the ED-TAS philosophy for GSM systems and showed that the attainable transmit diversity order is the same as that of the SM system employing ED-TAS. Furthermore, the BER performance of the ED-TAS aided GSM system was shown to be significantly better than that of its counterpart operating without ED-TAS. Additionally, we have shown that the minimum ED in case of the SM system employing ED-TAS does not change, when the size of the signal set is increased beyond a certain point, which enables low-complexity implementation of ED-TAS for large signal sets. Furthermore, it would be interesting to study the performance of i) ED-TAS aided GSM operating with erroneous feedback channel; ii) ED-TAS aided GSM in the frequency selective channel; iii) low-complexity TAS schemes of GSM.

\section{REFERENCES}

[1] R. Mesleh, H. Haas, S. Sinanovic, C. Ahn and S. Yun "Spatial modulation," IEEE Trans. Veh. Technol., vol. 57, no. 4, pp. 2228-2242, July 2008.

[2] M. Di Renzo, H. Haas and P. M. Grant, "Spatial modulation for multipleantenna wireless systems - A Survey," IEEE Commun. Magazine, vol. 49, no. 12, pp. 182-191, Dec. 2011.

[3] J. Wang, S. Jia and J. Song, "Generalised spatial modulation system with multiple active transmit antennas and low complexity detection scheme," IEEE Trans. Wireless Commun., vol. 11, no. 4, pp. 1605-1615, April 2012.

[4] R. Rajashekar, K.V.S. Hari, L. Hanzo, "Reduced-complexity ML detection and capacity-optimized training for spatial modulation systems," IEEE Trans. Commun., vol. 62, no. 1, pp. 112-125, Jan. 2014.

[5] P. Yang, M. Di Renzo, Y. Xiao, S. Li and L. Hanzo, "Design guidelines for spatial modulation," IEEE Communications Surveys \& Tutorials, vol. 17, no. 1, pp. 6-26, First quarter 2015.

[6] N. Ishikawa, R. Rajashekar, S. Sugiura and L. Hanzo, "Generalizedspatial-modulation-based reduced-RF-chain millimeter-wave communications," IEEE Trans. Veh. Technol., vol. 66, no. 1, pp. 879-883, Jan. 2017.
[7] P. Yang, Y. Xiao, Y. L. Guan, Z. Liu, S. Li and W. Xiang, "Adaptive SMMIMO for mmWave communications with reduced RF chains," IEEE J. Sel. Areas Commun.. vol. 35, no. 7, pp. 1472-1485, Jul. 2017.

[8] P. Wolniansky, G. Foschini, G. Golden and R. Valenzuela, "V-BLAST: an architecture for realizing very high data rates over the rich-scattering wireless channel," in Proc. International Symp. Signals, Syst., Electron., Pisa, Italy, pp. 295-300, Sep. 1998.

[9] E. Basar, U. Aygolu, E. Panayirci and H. V. Poor, "Space-time block coding for spatial modulation," IEEE Trans. Commun., vol. 59, no. 3 , pp. 823-832, Mar. 2011.

[10] R. Rajashekar and K.V.S. Hari, "Modulation diversity for spatial modulation using complex interleaved orthogonal design," in Proc. IEEE TENCON 2012, pp. 1-6, Nov. 2012.

[11] X. Li and L. Wang, "High rate space-time block coded spatial modulation with cyclic structure," IEEE Commun. Lett., vol. 18, no. 4, pp. 532-535, Apr. 2014

[12] A. G. Helmy, M. Di Renzo and N. Al-Dhahir, "Enhanced-reliability cyclic generalized spatial-and-temporal modulation," IEEE Commun. Lett., vol. 20, no. 12, pp. 2374-2377, Dec. 2016.

[13] P. Yang, Y. Xiao, L. Li, Q. Tang, Y. Yu and S. Li, "Link adaptation for spatial modulation with limited feedback," IEEE Trans. Veh. Technol., vol. 61, no. 8, pp. 3808-3813, Oct. 2012.

[14] R. Rajashekar, K.V.S. Hari and L. Hanzo, "Antenna selection in spatial modulation systems," IEEE Commun. Lett., vol. 17, no. 3, pp. 521-524, Mar. 2013.

[15] R. Rajashekar, K.V.S. Hari, K. Giridhar and L. Hanzo, "Performance analysis of antenna selection algorithms in spatial modulation systems with imperfect CSIR,' European Wireless 2013; 19th European Wireless Conference, Guildford, UK, pp. 1-5, 2013.

[16] Z. Zhou, N. Ge and X. Lin, "Reduced-complexity antenna selection schemes in spatial modulation," IEEE Commun. Lett., vol. 18, no. 1, pp. 14-17, Jan. 2014.

[17] N. Wang, W. Liu, H. Men, M. Jin, and H. Xu, "Further complexity reduction using rotational symmetry for EDAS in spatial modulation," IEEE Commun. Lett., vol. 18, no. 10, pp. 1835-1838, Oct. 2014.

[18] R. Rajashekar, K.V.S. Hari and L. Hanzo, "Quantifying the transmit diversity order of Euclidean distance based antenna selection in spatial modulation," IEEE Signal Proc. Lett., vol. 22, no. 9, pp. 1434-1437, Sep. 2015

[19] Z. Sun, Y. Xiao, L. You, L. Yin, P. Yang and S. Li, "Cross-entropy-based antenna selection for spatial modulation," IEEE Commun. Lett., vol. 20, no. 3, pp. 622-625, Mar. 2016.

[20] P. Yang, Y. Xiao, Y. L. Guan, S. Li and L. Hanzo, "Transmit antenna selection for multiple-input multiple-output spatial modulation systems," IEEE Trans. Commun., vol. 64, no. 5, pp. 2035-2048, May 2016.

[21] Z. Sun et al., "Transmit antenna selection schemes for spatial modulation systems: search complexity reduction and large-scale MIMO applications," IEEE Trans. Veh. Technol., vol. 66, no. 9, pp. 8010-8021, Sep. 2017.

[22] Y. Naresh and A. Chockalingam, "On media-based modulation using RF mirrors," IEEE Trans. Veh. Technol., vol. 66, no. 6, pp. 4967-4983, Jun. 2017.

[23] R. Rajashekar, M. Di Renzo, K.V.S. Hari and L. Hanzo, "A generalised transmit and receive diversity condition for feedback assisted MIMO systems: Theory \& applications in full-duplex spatial modulation," IEEE Trans. Signal Proc., vol. 65, no. 24, pp. 6505-6519, Dec. 15, 2017.

[24] R. Rajashekar, K.V.S. Hari and L. Hanzo, "Transmit antenna subset selection for single and multiuser spatial modulation systems operating in frequency selective channels," IEEE Trans. Veh. Technol, vol. 67, no. 7, pp. 6156-6169, July 2018.

[25] F. Shu et. al, "Two high-performance schemes of transmit antenna selection for secure spatial modulation," IEEE Trans. Veh. Technol., vol. 67, no. 9, pp. 8969-8973, Sept. 2018.

[26] Y. He, S. Atapattu, C. Tellambura and J. S. Evans, "Opportunistic group antenna selection in spatial modulation systems," to appear in IEEE Trans. Commun.

[27] Y. He, S. Atapattu, J. S. Evans and C. Tellambura, "A novel and tractable antenna selection in spatial modulation systems," in Proc. IEEE International Conference on Communications (ICC), pp. 1-6, 2018.

[28] H. Zhang, H. Dai, Q. Zhou and B. L. Hughes, "On the diversity order of spatial multiplexing systems with transmit antenna selection: A geometrical approach," IEEE Trans. Inf. Theory, vol. 52, no. 12, pp. 5297-5311, Dec. 2006.

[29] G. H. Golub, "Matrix computations," Johns Hopkins University, 1983. 